\title{
High Performance Dual-gate Dual-layer Amorphous Oxide Semiconductors TFTs on PI Foil for Display Application
}

\author{
Manoj Naga, Hikmet Celiker ${ }^{a, b}$, Lynn Verschueren ${ }^{a, b}$, Steve Smout ${ }^{a}$, Myriam Willegems ${ }^{a}$, \\ Rishabh Upadhyay c, Cédric Rolina, Nikolaos Papadopoulos ${ }^{a}$, Jan Genoe ${ }^{a, b}$, \\ Wim Dehaene $e^{b, a}$, Soeren Steudel ${ }^{a}$, Paul Heremans ${ }^{a, b}$ and Kris Myny ${ }^{a}$
}

\author{
a imec, Kapeldreef 75, 3001 Leuven, Belgium, Tel: +32 1628 1700, E-mail: manoj.nag @imec.be \\ ${ }^{\mathrm{b}}$ ESAT, Katholieke Universiteit Leuven, Kasteelpark Arenberg 10, 3001 Leuven, Belgium \\ ${ }^{c}$ AMITY University, Noida 201313, India
}

\begin{abstract}
We present dual-gate (DG) dual-layer (a-ITZO and a-IGZO) amorphous oxide TFTs integrated on polyimide (PI) foil. The fabricated TFTs show an apparent dual-gate field-effect mobility $\left(\mu_{F E}\right)$ of $45.0 \mathrm{~cm}^{2} /(V . s)$, sub-threshold slope $\left(S S^{-1}\right)$ of 0.40 V/decade and Ion/IofF ratio of $>10^{8}$. The DG dual-layer TFT based ring-oscillators exhibit faster oscillation frequencies ( $f_{\text {osc }}$ ) compared to DG a-IGZO TFT based ringoscillators. These TFTs are paving the way to faster scan drivers and higher resolution displays. It is also verified that with DG dual-layer TFTs, the current variation can be reduced when applying an off-panel compensation method to the asymmetric channel.
\end{abstract}

Keywords: Metal-oxide semiconductors; TFT; flexible displays

\section{Introduction}

Amorphous oxide semiconductors (AOS) such as IndiumGallium-Zinc-Oxide (IGZO), Silicon-Indium-Zinc-Oxide (SIZO), Hafnium-Indium-Zinc-Oxide (HIZO) and Zinc-Oxide (ZO) based thin-film transistors (TFTs) have shown desirable electrical properties, such as $\mu_{\mathrm{FE}}$ over $10.0 \mathrm{~cm}^{2} / \mathrm{Vs}$, high IoN $/$ IofF ratio and $\mathrm{SS}^{-1}$ below $0.30 \mathrm{~V} /$ decade and are thus potential candidates for device applications such as backplanes in active matrix displays (AMLCDs and AMOLEDs), large area imagers, and thin film circuits [1-6]. Particularly, the amorphous IGZO TFTs are already in volume manufacturing in flat-panel-display industry for AMLCD and AMOLED display backplanes. However, further improvement of TFT performance in terms of $\mu_{\mathrm{FE}}$ and $\mathrm{SS}^{-1}$ is required for higher resolution $(8 \mathrm{k} 4 \mathrm{k})$ display backplanes, the Gate-In-Panel (GIP) circuits in those display backplanes, low power circuit applications and FEOL/BEOL devices in CMOS

Few integration approaches have been made to achieve these high performance TFTs. In the first approach, several high mobility oxide materials such as Zinc-Tin-Oxide (ZTO), TinGallium-Zinc-Oxide (TGZO), Indium-Gallium-Oxide (IGO), Indium-Zinc-Tin-Oxide (ITZO), Aluminum-Zinc-Indium-TinOxide (AZITO), Indium-Zinc-Oxide (IZO), In-rich IndiumGallium-Zinc-Oxide (IGZO) and Zinc-Oxynitride ( $\mathrm{ZnON})$ have been reported with higher $\mu_{\mathrm{FE}}$ over $20.0 \mathrm{~cm}^{2} /(\mathrm{V} . \mathrm{s})$ while maintaining high IoN /IOFF ratio and steeper $\mathrm{SS}^{-1}$ [2-5]. In the second approach, by combining two material systems such as IZO and IGZO, ITO and IGZO, IZO and ITZO, different compositions of HIZO and many more, high performance TFTs with $\mu_{\mathrm{FE}}$ over $30.0 \mathrm{~cm}^{2} /$ (V.s) are reported [6-7]. Furthermore, in the third approach, the active semiconductor channel control

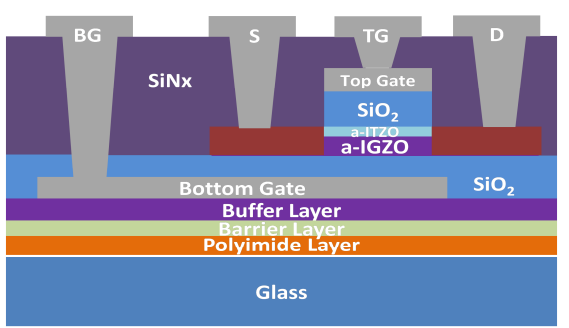

Figure 1. Cross-sectional image of DG dual-layer TFTs.

by dual-gate (DG) is reported with an apparent $\mu_{\mathrm{FE}}$ over 20.0 $\mathrm{cm}^{2} /(\mathrm{V} . \mathrm{s})$, steeper $\mathrm{SS}^{-1}$ and high IoN/IOFF ratio.

In this study, we have combined the advantages of dual-layer and DG control to enhance the performance of the amorphous oxide based TFTs without changing the integration flow of aIGZO layer based TFTs [7-10]. The dual-layer (a-ITZO and aIGZO) channel under DG control resulted in an apparent DG $\mu_{\mathrm{FE}}$ of over $45.0 \mathrm{~cm}^{2} /(\mathrm{V} . \mathrm{s}), \mathrm{SS}^{-1}$ of $0.40 \mathrm{~V} /$ decade and IoN $/$ IoFF ratio of $>10^{8}$. In order to investigate the speed of scan/line drivers, high performance ring oscillators are realized with the proposed DG dual-layer based TFTs and compared to ringoscillators integrated using DG a-IGZO TFTs. In addition, it is observed that the asymmetric semiconductor channel does not impose any problems with current compensation methods for AMOLED and AMLED panels.

\section{TFT Fabrication}

In the first step, a barrier film was deposited on PI foil. Subsequently, a buffer layer of $\mathrm{PECVD} \mathrm{SiO}_{2}$ is deposited on the a $15-\mu \mathrm{m}$ thick spin-on polyimide substrate. On the top of the buffer layer, a bottom gate-metal (MoCr) is deposited and patterned. This is followed by deposition of the back gate dielectric $\left(\mathrm{SiO}_{2}\right)$. After the gate-dielectric deposition, the semiconductor layer (dual-layer or a-IGZO) is deposited and patterned. These semiconductor layers are deposited using direct-current (DC) sputtering from ceramic targets of a-IGZO $(1: 1: 1)$ and a-ITZO $(\operatorname{In}(44 \%) / \operatorname{Sn}(12 \%) / \mathrm{Zn}(44 \%) / \mathrm{Al}(1 \%))$. In the next steps, the front gate-dielectric layer $\left(\mathrm{PECVD} \mathrm{SiO}_{2}\right)$ and the 
front gate-metal layer have been deposited. This is followed by the front gate-stack (Mo metal and $\mathrm{SiO}_{2}$ dielectric) patterning using a dry etch process. Post gate-stack patterning, an ICP CVD $\mathrm{SiNx}$ layer is deposited as an interlayer dielectric, followed by opening of the contact vias using a dry-etch process. Next, the source/drain ( $\mathrm{S} / \mathrm{D})$ metal $(\mathrm{Ti} / \mathrm{Al} / \mathrm{Ti})$ is integrated using lift-off process. Finally, the TFTs are annealed at $165^{\circ} \mathrm{C}$ in $\mathrm{N}_{2}$ environment for 3 hours. The electrical characteristics of the TFTs were measured using a parameter analyzer (Agilent 4156).

\section{Result and Discussion}

A schematic cross-section view of the DG dual-layer TFTs are shown in Fig. 1. In the self-aligned (SA) TFT configuration, the sheet resistivity (RSHEET) of the spacing region (SP) between gate and $\mathrm{S} / \mathrm{D}$ is responsible for limiting the transconductance and hence the $\mu_{\mathrm{FE}}$ of the TFTs [13-20]. An R RHEET of $<1.0$ $\mathrm{k} \Omega / \square$ is required for the SP region in order to maintain a channel resistance (RCHANNEL) higher than contact resistance (RCONTACT). The SP region is doped during the $\mathrm{SiNx}$ interlayer deposition due to hydrogen diffusion (impact of $\mathrm{SiH}_{4} / \mathrm{N}_{2}$ plasma) into AOS layers [18-20]. Since the RSHEET is inversely proportional to thickness of the AOS layer, a thicker AOS layer is needed to reduce the RSHEET. However, a thicker AOS layer reduces the $\mathrm{R}_{\text {CHANNEL }}$ as well, making it difficult to deplete the channel for $\mathrm{V}_{\mathrm{GS}}$ value close to zero volts. An integration change, such as an increase in thermal budget or an increase in $\mathrm{O}_{2}$ content while AOS deposition is then needed to avoid any change in the $R_{\text {ChANNEL }}[18,20]$. These changes may lead to other changes in the overall TFT integration. Therefore, in this work, we suggest a dual-layer approach to reduce the RSHEET of SP region while maintaining a large enough $\mathrm{R}_{\text {CHANNEL }}$.

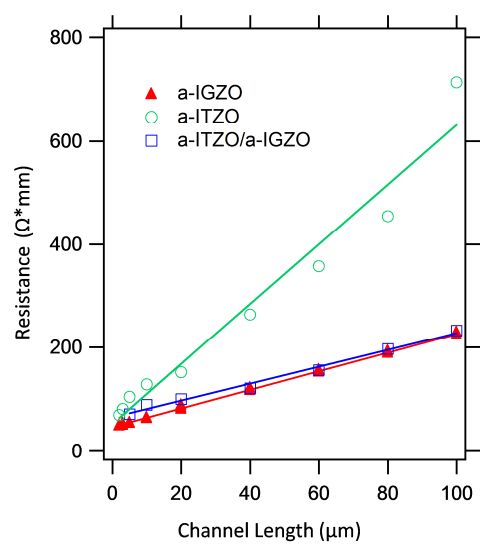

Figure 2. Comparison of the transistor resistance as a function of channel length ' $L$ ' for different TFTs with SiN $x$ interlayer. The contact resistances are obtained from the extrapolation to a zero-channel length.

To represent the impact of doping, another important parameter is the contact resistance (RCONTACT). RsheET and RCONTACT are extracted using transfer length method (TLM) method. The TLM structure contains nine resistive structures with fixed channel width $(\mathrm{W}=250 \mu \mathrm{m})$ and varying channel length $(\mathrm{L}=10$ $-100 \mu \mathrm{m})$ values. From the width normalized contact resistance 'RContact.W' versus channel length ' $L$ ' plot as shown in Fig. 2, the RSHEET and R this work are also compared with earlier published work on aIGZO and a-ITZO layers based TFTs. It is observed that a comparable value of RSHEET equal to $1.10 \mathrm{k} \Omega / \square$ is measured for both a-IGZO and dual-layer stack and it is close to earlier published work with a-IGZO layer [19, 20]. However, the 'RSHEET' is much lower compared to the a-ITZO layer based
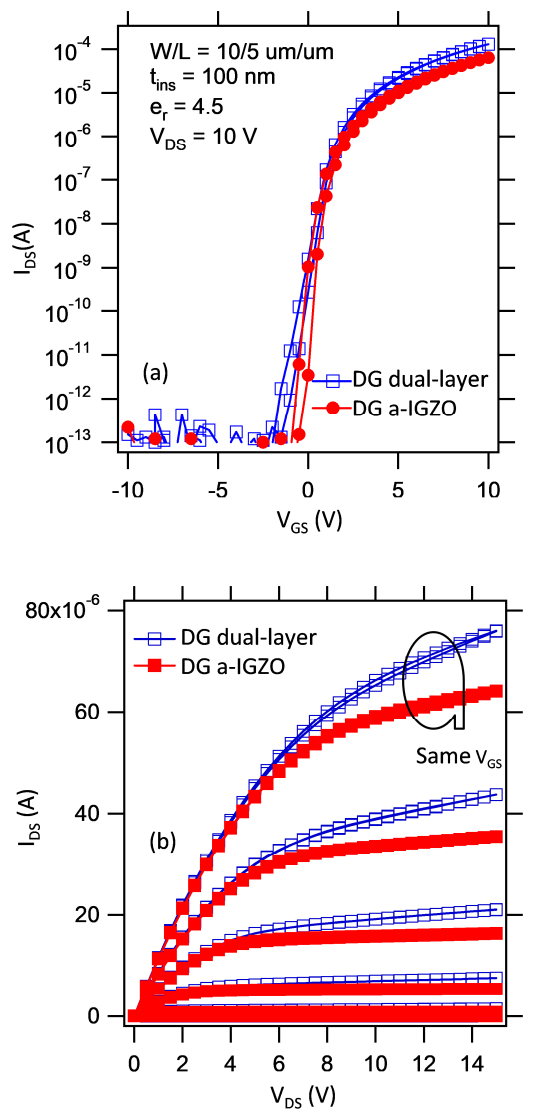

Figure 3. (a) Transfer ( $\left.V_{G S}-I_{D S}\right)$ and (b) output ( $\left.V_{D S}-I_{D S}\right)$ characteristics of TFTs on PI foil.

Table 1. Characteristics different TFT types.

\begin{tabular}{|l|c|c|c|c|}
\hline \multirow{2}{*}{ TFT Stack } & \multicolumn{3}{|c|}{ Parameters } & \multirow{2}{*}{ Ref. } \\
\cline { 2 - 4 } & $\begin{array}{c}\mu_{\mathrm{FE}} \\
\left(\mathrm{cm}^{2} / \mathrm{V} . \mathrm{s}\right)\end{array}$ & $\begin{array}{c}\mathrm{V}_{\mathrm{ON}} \\
(\mathrm{V})\end{array}$ & $\begin{array}{c}\mathrm{SS}^{-1} \\
(\mathrm{~V} / \mathrm{dec})\end{array}$ & \\
\hline SA a-IGZO TFTs & 12.0 & -1.0 & 0.50 & 17 \\
\hline DG a-IGZO TFTs & 20.0 & 0 & 0.28 & 19 \\
\hline SA a-ITZO TFTs & 27.0 & -1.5 & 0.40 & 20 \\
\hline DG a-IGZO TFTs & 20.0 & 0 & 0.28 & \multirow{2}{*}{ This } \\
\hline DG dual-layer TFTs & 45.0 & -1.0 & 0.40 & Work \\
\hline
\end{tabular}

TFT $(5.0 \mathrm{k} \Omega / \square)$, where the difference could be due to the thin ( 8 nm) a-ITZO layer [20]. Furthermore, comparable 'RCONTACT.W' (extracted by extrapolating the lines to the zero-channel length) values of $52.0 \Omega * \mathrm{~mm}$ and $57.0 \Omega * \mathrm{~mm}$ are obtained for a-IGZO and dual-layer based stacks, respectively.

Fig. 3(a) shows typical transfer characteristics of the DG duallayer and DG a-IGZO TFTs while applying $10 \mathrm{~V} \mathrm{~V}_{\text {DS. Figures of }}$ merit of these TFTs and a comparison to earlier published work 
is also summarized in Table 1. A trend in the $\mu_{\mathrm{FE}}$ improvement around different AOS based TFTs can be observed. In direct comparison to DG a-IGZO TFTs, the DG dual-layer TFTs exhibit about two times larger apparent DG $\mu_{\mathrm{FE}}$ of 45.0 $\mathrm{cm}^{2} /(\mathrm{V} . \mathrm{s})$, slightly negative $\mathrm{V}_{\mathrm{ON}}\left(\mathrm{V}_{\mathrm{TH}}\right)$ and comparable $\mathrm{SS}^{-1}$. Furthermore, output characteristics are shown in Fig. 3(b), displaying the expected larger on-currents of the DG dual-layer TFTs compared to DG a-IGZO TFTs for similar value of $\mathrm{V}_{\mathrm{GS}}$. Other characteristics such as clear pinch-off voltage and saturation at a low VDS voltage are comparable among these TFTs.

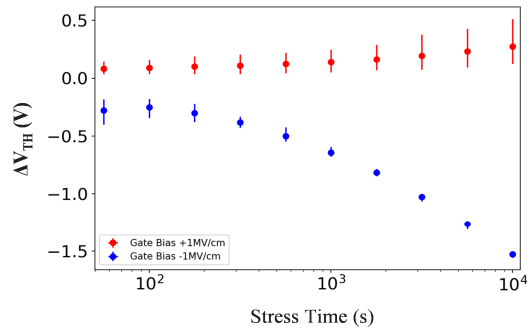

Figure 4. $\mathrm{V}_{\mathrm{TH}}$ shift as function of stress time under +/$1 \mathrm{MV} / \mathrm{cm}$ in dark at room temperature.

The bias stress shift behavior as a function of the stress time is also studied for these DG dual-layer TFTs. Under dark conditions and at room temperature, 12 TFTs ( $\mathrm{W}=15 \mu \mathrm{m}, \mathrm{L}=5$ $\mu \mathrm{m}$, and $\mathrm{SP}=10 \mu \mathrm{m}$ ) were stressed, 6 TFTs in the positive direction and 6 TFTs in the negative direction at a field strength of $+1 \mathrm{MV} / \mathrm{cm}\left(\mathrm{V}_{\mathrm{GS}}=+10 \mathrm{~V}\right.$ and $\left.\mathrm{V}_{\mathrm{DS}}=0 \mathrm{~V}\right)$ and $-1 \mathrm{MV} / \mathrm{cm}$ $\left(\mathrm{V}_{\mathrm{GS}}=-10 \mathrm{~V}\right.$ and $\left.\mathrm{V}_{\mathrm{DS}}=0 \mathrm{~V}\right)$, respectively for a period of $10^{4}$ seconds. It can be seen from Fig. 4 that a $\mathrm{V}_{\mathrm{TH}}$ change of less than $0.5 \mathrm{~V}$ is observed for positive gate bias and less than $-1.5 \mathrm{~V}$ for negative gate bias. These time-dependent $\mathrm{V}_{\mathrm{TH}}$ shift values are comparable to earlier published work on a similar type of TFT architecture $[17,19]$.

\section{Applicability for displays}

The higher mobility of the DG dual-layer TFTs may result in numerous advantages for display applications, in terms of brightness and/or resolution. Higher brightness displays can be achieved by replacing the semiconductor to the dual-layer, even without changing the pixel layout and driving voltages, due to the increased current. On the other hand, as the DG dual-layer TFTs yield larger currents, the driving voltage and supply voltage of the panel can be reduced, leading to a lower power consumption for the same brightness values. In terms of pixel scaling for AR/VR applications, a smaller footprint of the TFT will maintain the same electrical characteristics of the standard TFT, enabling higher pixel densities.

The higher mobility TFT integration flow will also be beneficial for integrated scan drivers, as a higher mobility semiconductor enables a stronger drive current for the same footprint, faster operating scan drivers to drive larger panels and optimize the scan driver for low power operation. This has been proven by realizing 19-stage ring oscillators based on the DG dual-layer TFTs and comparing it to DG a-IGZO TFTs [21]. The schematic of the Pseudo-CMOS inverter topology processed with different DG TFTs can be seen in Fig. 5 (a). Fig. 5 (b) depicts the measured oscillation frequency $\left(\mathrm{f}_{\mathrm{osc}}\right)$ with respect to supply voltage $\left(\mathrm{V}_{\mathrm{DD}}\right)$. Note that the stage delay $\left(\mathrm{t}_{\mathrm{d}}\right)$ of an inverter is calculated as $t_{d}=1 /\left(2 \times 19 \times f_{\text {osc }}\right)$. The VBIAS of this topology has been kept at double $V_{\text {DD. }}$ As seen from the graph (Fig. 5 (b)), the ring-oscillators based on DG dual-layer TFTs result faster frequencies compared to DG a-IGZO TFTs. Furthermore, Fig. 5(c) plots the ratio between the different oscillation frequencies. It can be concluded that the $f_{\text {osc }}$ ratio between ringoscillators based on DG dual-layer and ring-oscillators based on DG a-IGZO TFTs is excessively larger for lower values of $V_{D D}$. However, when $V_{D D}$ value is increased, the $f_{\text {osc }}$ ratio is reduced. For lower values of $\mathrm{V}_{\mathrm{DD}}$ (where $\mathrm{V}_{\mathrm{TH}}$ dominates), due to lower $\mathrm{V}_{\mathrm{TH}}$ and higher apparent $\mu_{\mathrm{FE}}$ (Fig. 3(a)), the fosc ratio of DG dual-layer TFTs based ring oscillators is excessively higher than DG a-IGZO TFTs based ring oscillators. However, when VDD value is increased, the value of $\mathrm{V}_{\mathrm{TH}}$ is being relatively less dominant and therefore the $f_{\text {osc }}$ ratio converges on a fixed value i.e. equal to apparent $\mu_{\mathrm{FE}}$ ratio.
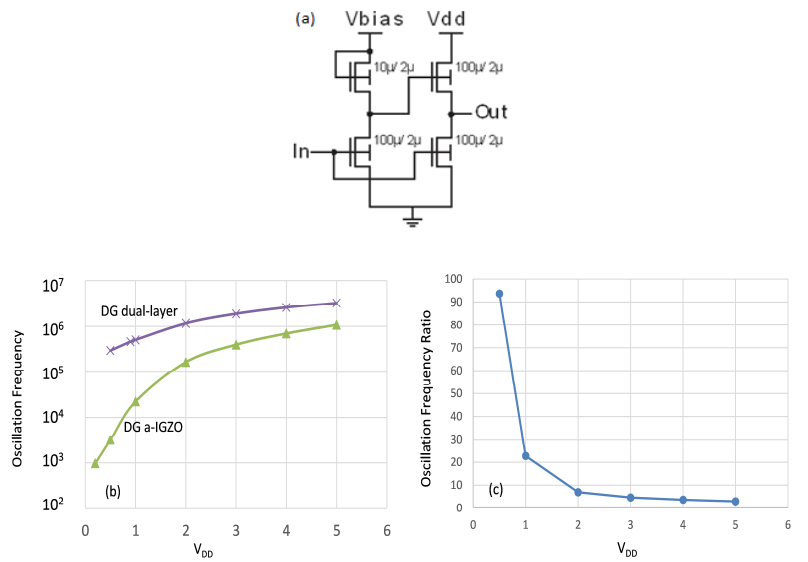

Figure 5. (a) Pseudo-CMOS inverter topology, (b) oscillation frequency with respect to $V_{D D}$ and (c) oscillation frequency ratio comparison with respect to $V_{D D}$.
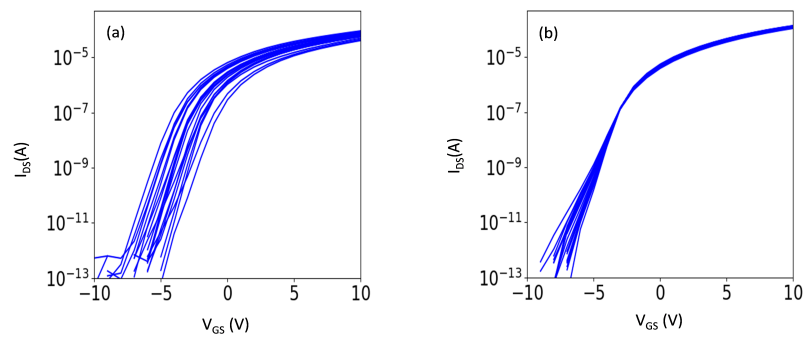

Figure 6. Measurement of current for different front-gate voltages of 21 TFTs (a) with a fixed back-gate voltage of $2 \mathrm{~V}$, (b) with the compensated back-gate voltage.

Another possible application of this DG dual-layer TFTs is the possibility to compensate for the TFT variations present in an AMOLED panel. Since the channel of this TFT comprises two layers of different semiconducting materials, the symmetry between the front-gate and the back-gate is reduced, adding a possible obstacle for previously reported calibration methods. To study the possibility for compensation with these DG duallayer TFTs, additional measurements have been performed, mimicking the compensation scheme proposed in [22].

Fig. 6 (a) shows the current measurements of 21 TFTs for different front-gate (data) voltages, with a fixed back-gate (calibration) voltage of $2 \mathrm{~V}$, corresponding to the current for the 
different pixels before calibration according to this method. After this measurement, the back-gate voltage is varied for a fixed front-gate voltage of $-3.0 \mathrm{~V}$, and the back-gate voltage that corresponds to a current of $137 \mathrm{nA}$ is extracted in the calibration cycle. Lastly, the current for different front-gate voltages, with the compensated back-gate voltage is measured and presented in Fig. 6 (b). From these measurements, the variation of the current at a certain front-gate (data) voltage can be calculated and it is observed that the variability can be reduced by a factor 48 with the calibration method. Before compensation, the current variation at a data voltage of $-3.0 \mathrm{~V}$ is $144 \%$, whereas after calibration the current variation at this data voltage is reduced to only $3.0 \%$. Therefore, we can conclude that the asymmetric TFT channel does not cause any problems for the calibration. Hence, the DG dual-layer TFT is highly suitable for display applications.

\section{Conclusion}

We have investigated the fabrication of high-performance DG dual-layer TFTs. The TFTs exhibit apparent DG $\mu_{\mathrm{FE}}$ of 45.0 $\mathrm{cm}^{2} /(\mathrm{V} \cdot \mathrm{s}), \mathrm{SS}^{-1}$ of $0.40 \mathrm{~V} / \mathrm{dec}$ and Ion $/$ IofF ratio of $>10^{8}$. In addition, we have evaluated a path for improved scan drivers, by demonstrating the DG dual-layer TFTs in 19-stage ringoscillators, resulting in larger oscillation frequencies compared to DG a-IGZO TFTs. Finally, we have been able to investigate the current compensation method for AMOLED panels, to strongly reduce the present variability of TFTs in pixels.

\section{Acknowledgements}

Part of this work has received funding from the European Research Council (ERC) under the European Union's Horizon 2020 research and innovation program under grant agreement No. 716426 (FLICs project).

\section{References}

[1] K. Nomura et al., "Amorphous Oxide Semiconductors for High-Performance Flexible Thin-Film Transistors", Nature 488, Vol. 432, 488-492, 2004.

[2] Y. Ye et al., "High mobility amorphous zinc oxynitride semiconductor material for thin film transistors," Journal of Applied Physics 106, 074512 (2009).

[3] M. K. Ryu et al., "Gate Insulator for High Mobility Oxide TFT," IEEE Electron Device Letters, 31 (2), 144 (2010).

[4] T. Arai et al., "Emergent oxide TFT technologies for next-generation AM-OLED displays," SID Int. Symp. Digest Tech. Papers, 710 (2011).

[5] K. Ebata et al., "High-Mobility Thin-Film Transistors with Polycrystalline In-Ga-O Channel Fabricated by DC Magnetron Sputtering," Applied Physics Express 5, 011102 (2012).

[6] A. D. Mourey et al., "Self-Aligned-Gate ZnO TFT Circuits," IEEE Electron Device Letters, Vol. 31, No. 4, p. 326-328, April 2010.

[7] G. Baek et al., "Electrical properties and stability of dual-gate coplanar homojunction DC sputtered amorphous indium-gallium-zinc-oxide thin-film transistors and its application to AM-OLEDs," IEEE
Trans. Electron Devices, 58, No. 12, 4344-4353, Dec. (2011).

[8] M. Mativenga et al, "Bulk accumulation a-IGZO TFT for high current and turn-on voltage uniformity," IEEE Electron Device Lett., 34, No. 12, 1533-1535, Dec. (2013).

[9] S. Zhang et al., "A novel self-aligned double-gate TFT technology," IEEE Electron Device Lett., 22, No. 11, 530-532, Nov. (2001).

[10] N. Münzenrieder et al., "Flexible self-aligned doublegate IGZO TFT," IEEE Electron Device Lett., 35, No. 1, 69-71, Jan. (2014).

[11] N. Morosawa, et al., "A Novel Self-Aligned Top-Gate Oxide TFT for AM-OLED Displays," Sony Corporation, 479482, SID 2011.

[12] S. Kim et al., "Source/Drain Formation of Self-Aligned Top-Gate Amorphous GaInZnO Thin-Film Transistors by $\mathrm{NH}_{3}$ Plasma Treatment," IEEE Electron Device Letters, Vol. 30, No. 4, April 2009, p. 374-376.

[13] B. D. Ahn et al., "Comparison of the effects of Ar and $\mathrm{H}_{2}$ plasmas on the performance of homo junctioned amorphous indium gallium zinc oxide thin film transistors," Appl. Phys. Lett., vol. 93, no. 20, p. 203 506, Nov. 2008.

[14] J. Park et al., "Self-aligned top-gate amorphous gallium indium zinc oxide thin film transistors," Appl. Phys. Lett., vol. 93, no. 5, p. 053501, Aug. 2008.

[15] C. H. Wu et al., "Self-aligned top-gate coplanar In-GaZn-O thin-film transistors," J. Display Technol., vol. 5, no. 12 , p. 515-519, Dec. 2009.

[16] D. H. Kang et al., "Self-aligned coplanar a-IGZO TFTs and application to high-speed circuits," IEEE Electron Device Lett., vol. 32, no. 10, p. 1385-1387, Oct. 2011.

[17] M. Nag et al., "Circuits and AMOLED display with self-aligned a-IGZO TFTs on polyimide foil," Journal of the Society for Information Display, Vol. 22, Issue 10, pp. 509-517, October 2014.

[18] S. Steudel et al., "Flexible AMOLED Display with Integrated Gate Driver Operating at Operation Speed Compatible with 4k2k," SID Symposium Digest of Technical Papers, 46, 2015.

[19] M. Nag et al., "Characteristics improvement of top-gate self-aligned amorphous indium gallium zinc oxide thinfilm transistors using a dual-gate control," Volume 25, Issue 6, Pages 349-355, June 2017.

[20] R. Upadhyay et al., "Self-Aligned Amorphous IndiumTin-Zinc-Oxide Thin Film Transistors on Polyimide Foil," ECS Journal of Solid State Science and Technology, 7 (4) P185-P191 (2018).

[21] K. Myny et al., "Unipolar Organic Transistor Circuits Made Robust by Dual-Gate Technology," IEEE Journal of Solid-State Circuits, Vol. 46, No. 5, May 2011, p. 1223.

[22] L. Verschueren et al., "40x Current Variation Reduction Enabled by an External $\mathrm{V}_{\mathrm{T}}$-Compensation Scheme for AMOLED Displays using a 3T2C-Pixel Circuit with Dual-Gate TFTs," SID Symposium Digest of Technical Papers, 49, 2018. 